\title{
Détermination de l'indice de fibrosité et certains de ses biais chez le mouton
}

\author{
AG Deswysen, C Audic, E Amouche \\ Université catholique Louvain, faculté des Sciences agricoles, unité de Génétique, \\ place Croix-du-Sud, 2 (boite 14), B-1348, Louvain-la-Neuve, Belgique
}

L'objectif de ce travail est de préciser l'importance de certains biais de mesures liés au niveau d'ingestion et à la présence d'activités masticatoires parasites sur la détermination de l'indice de fibrosité caractérisé par l'activité masticatoire totale $(\mathrm{min} / \mathrm{kg}$ MS).

Six moutons mâles castrés, de race lle-deFrance, âgés de 18 mois $(68,2 \mathrm{~kg})$, ont reçu selon un schéma expérimental en crossover $3 x$ $3 \times 6(3 \times 24$ j) 3 types de rations : 1) foin ad libitum ( $F ; 59,0 \% \mathrm{NDF}$ ); 2) foin en quantité limitée à $70 \%$ de l'ad libitum (FQL); 3) concentré $(90 \%$; $41,4 \%$ NDF) et foin $(10 \%)$ en quantité limitée égale à $70 \%$ de l'ad libitum foin (CQL). Les mouvements de mâchoires furent enregistrés en continu durant les 8 derniers jours de chaque période expérimentale. Additionnellement pour CQL, la fin des 2 repas journaliers de concentré et de foin fut observée visuellement et toutes autres activités de type ingestif enregistrées (en observation visuelle : léchage, mordillement des auges...) furent classées en activités parasites.

L'indice de fibrosité a été plus élevé $(P<$ 0,05 ) pour $F Q L$ que pour $F$, confirmant ainsi les observations de Luginbuhl et al (1989) mais non celles d'Andrieu et al (1987). Pour CQL, la prise en considération des activités parasites comme activités masticatoires de type ingestif a entraîné une large surestimation de l'indice de fibrosité. Cela suggère la nécessité d'une grande prudence quant à l'utilisation de valeurs d'indice de fibrosité, spécialement celles déterminées dans des régimes riches en concentrés.

Andrieu J, Demarquilly C, Korea A (1987) $R e$ prod Nutr Dév 27 (1B), 189-190

Luginbuhl JM, Pond KR, Burns JC, Russ JC (1989) J Anim Sci 67, 3410-3418

Tableau I. Ingestion journalière (I), durée journalière de mastication (DM) et indice de fibrosité (IF) mesurés sur 6 moutons.

\begin{tabular}{lccccc}
\hline Paramètres & $F$ & $F Q L$ & CQL & $E S$ & Valeur P \\
\hline I (g MS/kg PV0,75/j) & $52,8^{\mathrm{a}}$ & $35,9^{\mathrm{b}}$ & $28,5^{\mathrm{b}}$ & 0,9 & $<0,001$ \\
$\begin{array}{l}\mathrm{DM}(\mathrm{min} / \mathrm{j}) \\
\quad \text { Totale }\end{array}$ & $881^{\mathrm{a}}$ & $712^{\mathrm{b}}$ & $427^{\mathrm{c}}$ & 21 & $<0,001$ \\
$\quad$ Sans les activités parasites & & & 297 & & \\
$\begin{array}{l}\text { IF (min/kg MS ingérée) } \\
\quad \text { Total }\end{array}$ & $695^{\mathrm{a}}$ & $831^{\mathrm{b}}$ & $479^{\mathrm{c}}$ & 21 & $<0,001$ \\
$\quad$ Sans les activités parasites & & & 329 & & \\
\hline
\end{tabular}

Les moyennes sur une même ligne qui n'ont pas une lettre en commun diffèrent $(P<0,05)$. 